\title{
Left Atrial Appendage Remodeling after Atrial Fibrillation Ablation
}

Yichi Zhang ${ }^{1}$, Abdel Hadi El Hajjar ${ }^{1}$, Chao Huang ${ }^{1}$, Aneesh Dhore-Patil ${ }^{1}$, Mario Mekhael ${ }^{1}$, Charbel Noujaim ${ }^{1}$, Lilas Dagher ${ }^{1}$, Alan Morris ${ }^{1}$, Chan Ho Lim ${ }^{1}$, Eugene Kholmovski ${ }^{1}$, Raman Danrad ${ }^{2}$, Tarek Ayoub ${ }^{1}$, Christopher Pottle ${ }^{1}$, and Nassir Marrouche ${ }^{1}$

${ }^{1}$ Tulane Medical Center

${ }^{2}$ Louisiana State University School of Medicine-New Orleans

January 6, 2022

\begin{abstract}
Introduction: Larger left atrial appendage (LAA) ostium area and greater left atrial (LA) volume have been associated with an increased risk of ischemic stroke. Catheter ablation (CA) of atrial fibrillation (AF) leads to morphological and functional changes within the LA and LAA, some of which are not well studied. Here, we present findings regarding post-ablation changes of the LAA ostia and correlate them with various LA, LAA and left ventricular (LV) functional and morphological metrics. Methods: This retrospective analysis included patients scheduled to undergo first-time radiofrequency CA for AF. Catheter ablation techniques included PVI with or without additional ablations. Cardiac magnetic resonance imaging (CMR) was used to assess LA, LAA and LV morphology and function, including LAA ostium area, LA/LAA volume and volume index, LA ejection fraction, LA strain, and LV ejection fraction. A Kruskal-Wallis test was used for correlating LAA ostial dimensions with other LA morphological and functional metrics. The t-test or two-sample Wilcoxon test were used to compare LA and LAA morphological parameters. Results: A total of 101 patients with AF were included in this study. The mean age was 60.1 \pm 11.1 years, $69 \%$ were male, the average BMI was $29.22 \pm 5.08$. The LAA ostial area reduced significantly from $3.84 \pm 1.15$ $\mathrm{cm}^{2}$ before ablation to $3.42 \pm 0.96 \mathrm{~cm}^{2}$ after ablation $(\mathrm{p}=0.0004)$. This reduction was asymmetrical, as the minor axis length decreased from $1.92 \mathrm{~cm}$ to $1.77 \mathrm{~cm}$ without significant changes in the major axis. LVEF increased from a pre-ablation average of $48.26 \%$ to a post-ablation average of $53.62 \%(\mathrm{p}=0.015)$. Correlation of pre-ablation LVEF and LAA ostium area showed a near-significant negative trend $(\mathrm{r}=-0.21, \mathrm{p}=0.083)$. LAEF correlated negatively with LAA ostial area $(\mathrm{r}=-0.289, \mathrm{p}=0.0057)$, total LA strain $(\mathrm{r}=-0.248, \mathrm{p}=0.0185)$, and passive LA strain $(\mathrm{r}=-0.208, \mathrm{p}=0.049)$. Conclusion: There is a significant asymmetrical reduction of the LAA ostial area after AF ablation that is independent of LVEF changes. Larger LAA ostial area was associated with lower LAEF and LA strain. Remodeling of the LAA after AF ablation may help account for reduced risk of stroke and increased cardiac function.
\end{abstract}

\section{Left Atrial Appendage Remodeling after Atrial Fibrillation Ablation}

Yichi Zhang $\mathrm{BS}^{1}$, Abdel Hadi El Hajjar $\mathrm{MD}^{1}$, Chao Huang $\mathrm{PhD}^{1}$, AneeshDhore-Patil $\mathrm{MD}^{1}$, Mario MekhaelMD ${ }^{1}$, Charbel Noujaim $\mathrm{MD}^{1}$, Lilas Dagher $\mathrm{MD}^{1}$, Alan Morris $\mathrm{MS}^{1}$, Chan Ho Lim $\mathrm{MS}^{1}$, EugeneKholmovski $\mathrm{PhD}^{1,2}$, Raman Danrad $\mathrm{MD}^{3}$, Tarek Ayoub $\mathrm{MD}^{1}$, Christopher Pottle $\mathrm{MS}^{1}$, Nassir Marrouche $\mathrm{MD}^{1, \#}$

${ }^{1}$ TRIAD Center, Tulane University School of Medicine, New Orleans, LA

${ }^{2}$ Department of Biomedical Engineering, Johns Hopkins University, Baltimore, MD

${ }^{3}$ Lousiana State University School of Medicine, New Orleans, LA

Corresponding Author: 
Nassir F. Marrouche, MD

Professor of Medicine,

Director Heart and Vascular Institute

Tulane University School of Medicine

1430 Tulane Ave, New Orleans, LA, 70112.

nmarrouche@tulane.edu

Abstract:

Introduction: Larger left atrial appendage (LAA) ostium area and greater left atrial (LA) volume have been associated with an increased risk of ischemic stroke. Catheter ablation (CA) of atrial fibrillation (AF) leads to morphological and functional changes within the LA and LAA, some of which are not well studied. Here, we present findings regarding post-ablation changes of the LAA ostia and correlate them with various LA, LAA and left ventricular (LV) functional and morphological metrics.

Methods : This retrospective analysis included patients scheduled to undergo first-time radiofrequency CA for AF. Catheter ablation techniques included PVI with or without additional ablations. Cardiac magnetic resonance imaging (CMR) was used to assess LA, LAA and LV morphology and function, including LAA ostium area, LA/LAA volume and volume index, LA ejection fraction, LA strain, and LV ejection fraction. A Kruskal-Wallis test was used for correlating LAA ostial dimensions with other LA morphological and functional metrics. The t-test or two-sample Wilcoxon test were used to compare LA and LAA morphological parameters.

Results : A total of 101 patients with AF were included in this study. The mean age was $60.1 \pm 11.1$ years, $69 \%$ were male, the average BMI was $29.22 \pm 5.08$. The LAA ostial area reduced significantly from $3.84 \pm$ $1.15 \mathrm{~cm}^{2}$ before ablation to $3.42 \pm 0.96 \mathrm{~cm}^{2}$ after ablation $(\mathrm{p}=0.0004)$. This reduction was asymmetrical, as the minor axis length decreased from $1.92 \mathrm{~cm}$ to $1.77 \mathrm{~cm}$ without significant changes in the major axis. LVEF increased from a pre-ablation average of $48.26 \%$ to a post-ablation average of $53.62 \%(\mathrm{p}=0.015)$. Correlation of pre-ablation LVEF and LAA ostium area showed a near-significant negative trend ( $\mathrm{r}=-0.21$, $\mathrm{p}=0.083)$. LAEF correlated negatively with LAA ostial area $(\mathrm{r}=-0.289, \mathrm{p}=0.0057)$, total LA strain $(\mathrm{r}=-0.248$, $\mathrm{p}=0.0185)$, and passive LA strain $(\mathrm{r}=-0.208, \mathrm{p}=0.049)$.

Conclusion: There is a significant asymmetrical reduction of the LAA ostial area after AF ablation that is independent of LVEF changes. Larger LAA ostial area was associated with lower LAEF and LA strain. Remodeling of the LAA after AF ablation may help account for reduced risk of stroke and increased cardiac function.

Keywords : Atrial Fibrillation, Left Atrial Appendage, Atrial Remodeling, Atrial Myopathy, Cardiac MRI

\section{Background:}

Atrial fibrillation (AF) is the most common arrythmia in the US, with an estimated prevalence of 1-2\% (1), which increases with age to $20 \%$ in patients in their $80 \mathrm{~s}$ (2). One of the most feared complications in AF patients is cardioembolic stroke (3), with studies suggesting case fatality rates of up to $27 \%$ (4). Thus, current guidelines for the treatment of AF involve a dual-strategy of rate/rhythm control for prevention of arrythmia, as well as oral anticoagulation (OAC) for mitigation of stroke risk (5). Prior data suggested that rate control was non-inferior to rhythm control (AFFIRM trial)(6), recently early rhythm control is proving to improve outcomes (EAST-AF, EARLY-AF) $(7,8)$. Catheter ablation of AF has proven to be a safe and effective strategy in drug-refractory AF patients. Recent trials have suggested that ablation is superior to medication for reducing mortality in certain subsets of AF patients (9) and that the former may also provide better quality-of-life (10). However, the effect of catheter ablation on LA/LAA morphology and function is poorly understood (11). 
The left atrium (LA) serves a complex "trio" of functions, working as a reservoir, conduit, and booster pump at different points of the cardiac cycle (12). The LA's relatively weaker contractility compared to the left ventricle (LV) increases the possibility of blood stasis (13). The left atrial appendage (LAA) is the most common source for the formation of systemic thromboemboli due to its unique shape and increased tendency for blood stasis (14). The entrance from the LA into the LAA is the LAA ostium, which is usually defined by the coumadin ridge superiorly and the left circumflex artery inferiorly (15). The LAA ostium was initially studied and measured for developing techniques for LAA occlusion (16), such as the use of ligation, clip or a Watchman device (17). However, detailed evidence is lacking on how baseline LAA ostium characteristics are correlated with other anatomical and clinical parameters of the LA and LAA in AF patients. Furthermore, there is a paucity of data on how the LAA ostium changes after AF ablation. These findings can help us better understand underlying mechanisms of LA/LAA morphological changes following catheter ablation.

In this study we aim to assess baseline LAA ostium characteristics among AF patients and correlate them with anatomical and functional parameters of the LA and LAA, as well as patients' preexisting comorbidities and risk factors. We will also be the first to report changes in the LAA ostium following AF catheter ablation.

\section{Methods:}

This is a retrospective observational study of AF patients who underwent catheter ablation at Tulane Medical Center. All patients were treated according to the current guidelines with rate/rhythm control and OAC. Patients who were scheduled for catheter ablation received pre-ablation cardiac magnetic resonance imaging (CMR) to ascertain LA fibrosis, LA and LAA anatomical and functional characteristics as well as left ventricular ejection fraction (LVEF). Catheter ablation techniques included PVI with or without additional ablations. Additional ablation lines were left to the discretion of the treating physician. A subset of patients who completed AF ablation also underwent another CMR study approximately three months to one year after their procedure to re-assess LA and LAA anatomical and functional parameters and associated changes postablation. All relevant baseline demographic information, past medical history, current clinical parameters, and imaging characteristics were collected and stored in a HIPAA compliant, secure database for analysis. The Tulane university institutional review board approved this study (IRB \#2019-1803).

\section{CMR Study Protocol:}

All patients underwent a CMR study on a 1.5 Tesla clinical MR scanner (Ingenia, Philips Healthcare). CMR protocol included Cine MRI of LA and LV, and 3D late gadolinium enhancement (LGE) scan. Cine MRI was performed in axial plane for LA and short axis plane for LV using a retrospective ECG-gated balanced steady-state free precision (bSSFP) pulse sequence. Cine scan for LA covers the whole LA and LAA by a stack of contiguous 2D axial slices with thickness of $7.0 \mathrm{~mm}$ and spatial resolution of $1.56 \times 1.74 \mathrm{~mm}$. The other scan parameters for this Cine scan were repetition time $(\mathrm{TR})=2.80 \mathrm{~ms}$, echo time $(\mathrm{TE})=1.40 \mathrm{~ms}$, flip angle $(\mathrm{FA})=60^{\circ}$. Cine scan of LV covers the whole LV by a stack of $2 \mathrm{D}$ short-axis slices with thickness of $8.0 \mathrm{~mm}$, inter-slice gap of $0.8 \mathrm{~mm}$, spatial resolution of $1.45 \times 1.65 \mathrm{~mm}$. The other scan parameters for Cine of LV scan were TR $=3.48 \mathrm{~ms}$, TE $=1.74 \mathrm{~ms}, \mathrm{FA}=90^{\circ}$. Scan protocol for 3D LGE was similar to 3D LGE protocol optimized and validated in DECAAF and DECAAF II multi-center studies (18). LGE scan was initiated 20-25 minutes after contrast agent administration using a 3D inversion recovery prepared, respiration navigated, ECG-gated, spoiled gradient echo pulse sequence. 3D LGE imaged axial slab covering LA and LAA with spatial resolution of $1.25 \times 1.25 \times 2.5 \mathrm{~mm}$. The other scan parameters for 3D LGE were TR $=5.26 \mathrm{~ms}, \mathrm{TE}=2.58 \mathrm{~ms}$, and $\mathrm{FA}=20^{\circ}$.

\section{LA Image Segmentation:}

Left atrial wall was manually segmented by expert observers from the LGE-MRI images using the Corview image processing software (MARREK, Salt Lake City, UT). Briefly, the protocol for segmentation proceeded as follows: first, the endocardial border of the left atrium was defined, including the PV extensions, by manually tracing the left atrium-PV blood pool in each slice of the LGE-MRI volume. Next, the endocardial segmentation was morphologically dilated and then manually adjusted to create an assessment of the boundary of the epicardial left atrium surface. Finally, the endocardial segmentation was subtracted from 
the epicardial segmentation to define a wall segmentation, which was manually edited to exclude the mitral valve and PVs. The resulting left atrial wall segmentation included the $3 \mathrm{D}$ extent of both the left atrial wall and the antral regions of the PVs. Images that could not be segmented or quantified due to poor contrast, blurred left atrial boundaries, and presence of artifacts in left atrial region were defined as poor quality.

\section{LA Fibrosis Assessment}

Quantification of LA fibrosis was obtained using methods previously described(19). The LA wall was segmented manually and regions of fibrosis in LGE-MRI images were defined by an intensity threshold determined by expert inspection. Fibrotic tissue is detected when its intensity is two to three standard deviations above the mean of the normal tissue intensity. A 3D LA fibrosis map was created using volume rendering module in Corview software (Marrek Inc., Salt Lake City, UT). Inter-observer and intra-observer reproducibility for this technique was previously reported (17). Fibrosis was represented as the volumetric percentage of left atrial wall enhancement.

\section{LA Morphological and Functional Parameters Assessment:}

A stack of contiguous axial cine images covering LA and LAA was acquired. Tissue-tracking approach was used to quantify LA strain and phasic volumes from the cine images. Endocardial and epicardial LA borders was defined at end-systole by a blinded operator. Using defined borders in each view, the software tracked the LA myocardium in subsequent frames. The operator validated the generated contours during the cardiac cycle for quality control. Images with poor tracking were excluded. In each frame, the LA volume was calculated. Maximum, minimum, and pre-atrial contraction LA volumes (LAVmax, LAVmin, VPreA) were extracted from volume curve. The point on the LA volume curve during diastole just before the fast descent following a period of stability in LA volume was defined as preatrial contraction volume. At that point, the atrial strain rate was equal to zero. All measured volumes were indexed to body surface area. The global longitudinal strain and strain rate were calculated by averaging the strain values over whole LA.

The volumetric LA function parameters were calculated as follows:

Total LA EF (in \%) $=[($ LAVmax $-\mathrm{LAVmin}) / \mathrm{LAVmax}] \times 100$

Active LAEF (in \%) $=[($ VPreA - LAVmin $) /$ VPreA $] \times 100$

Passive LAEF (in \%) $=[$ LAVmax - VPreA)/LAVmax $] \times 100$

\section{LAA Morphological and Functional Parameter Assessment}

The morphology of the LAA was assessed from 3D LGE images using particle-based modeling (PBM) for statistical shape analysis (SSA). Briefly, after contouring and segmentation by trained observers, we created a three-dimensional reconstruction of the LAA. SSA using particle-based modeling was performed on the LAA reconstruction to define the shape class. For LAA ostium measurements, we used three planes on the CMR to define the ostium and estimate its maximal diameter, minimal diameter, circumference, and surface area. The ostium of the LAA was first defined on the transversal image by the plane that connected between the base of the Coumadin ridge and the proximal left circumflex artery. The cross-section of the LAA ostium was created from orthogonal projections in the coronal plane. The maximum and minimum LAA ostium diameters were measured from the sagittal image. The circumference and surface area were then calculated and provided by the software. Figure 1 shows the procedural steps for registration of the pre- and post-ablation LGE-MRI images to ensure spatial alignment as well as for measurement of the LAA ostium.

\section{Statistical Analysis}

All statistical tests were two-sided with p[?]0.05. Descriptive statistics such as mean (SD) for continuous variables and frequency (\%) for categorical variables were reported for the study sample. Statistical analysis was performed using SAS version 9.4 and STATA version 14. A Kruskal-Wallis test was used for correlating LAA ostial measurements with other LA morphological and functional metrics. The t-test or two-sample 
Wilcoxon test were used to compare LA and LAA morphological parameters in patients with or without certain comorbidities, depending on normality of variable. The two-sample Wilcoxon test was also used to compare pre- and post-ablation LAA ostial parameters.

Results:

\section{Baseline Characteristics:}

A total of 101 patients with AF were included in the study. The mean age was $60.1+-11.1$ years, $69 \%$ were male, the average BMI was $29.22+-5.08$ and the average body surface area (BSA) was $1.83+-0.68$ $\mathrm{m}^{2}$. Of all patients, $23.8 \%$ had heart failure with reduced ejection fraction (HFrEF), $6.9 \%$ had heart failure with preserved ejection fraction (HFpEF), $46.5 \%$ had hypertension (HTN), and $7.9 \%$ had a history of stroke or other systemic thromboembolism (SSE). $33.7 \%$ of patients underwent some form of cardioversion and $38.6 \%$ had failed a trial of antiarrhythmic therapy. The average left ventricular ejection fraction (LVEF) was $48.85 \%$.

In terms of baseline left atrial characteristics, the mean LAVI was $38.66+-10.2 \mathrm{~mL} / \mathrm{m}^{2}$, the mean LA sphericity index was $73.6+-18.9$, the mean LAA volume was $6.76+-4.47 \mathrm{~mL}$, and the mean fibrosis level was $15.51+-8.02 \%$. Left atrial appendage shapes were categorized into the popular "windsock" (6.93\%), "cactus" $(5.94 \%)$, "chicken wing" $(77.2 \%)$ and "cauliflower" (9.90\%) categories (20). The baseline characteristics of the LA, LAA, and LAA ostia in our study cohort are given in Table 1.

\section{LAA Ostium Dimensions}

The average LAA ostium area was $3.32+-1.17 \mathrm{~cm}^{2}$. When accounting for BSA, the indexed mean LAA ostium area was $1.67+-0.56 \mathrm{~cm}^{2} / \mathrm{m}^{2}$. Since LAA ostia can be approximated with a centroid with a major and minor axis, the average major axis length was $2.44+-0.48 \mathrm{~cm}$, the average minor axis length was 1.72 $+-0.34 \mathrm{~cm}$, with the average perimeter at $6.35+-1.17 \mathrm{~cm}$.

When exploring the relationship between the LAA ostia characteristics and various risk factors and comorbidities, patients with HFrEF had a significantly larger LAA ostial area than patients without HFrEF (3.88 vs. $3.20 \mathrm{~cm}^{2}$, respectively, $\left.\mathrm{p}=0.0148\right)$. This result remained significant when comparing the LAA ostial area indexed by BSA ( 1.89 vs. $1.60 \mathrm{~cm}^{2} / \mathrm{m}^{2}$, respectively, $\mathrm{p}=0.0268$ ). No statistically significant associations were found between LAA ostial area and other risk factors or comorbidities. The analysis results are presented in Table 2 .

\section{Left Atrial Appendage Ostium Dimensions and its Association with Left Atrial Morphological and Functional Parameters}

The LAA ostial parameters were further examined for their correlation with LA and LAA morphological parameters. The LAA ostial area was shown to have a moderate positive correlation with LA volume $(\mathrm{r}=0.31, \mathrm{p}=0.0017)$ and LAA volume $(\mathrm{r}=0.42, \mathrm{p}<0.0001)$. LAA ostial area was largest in patients with a "cauliflower" LAA shape morphology $\left(4.53 \mathrm{~cm}^{2}\right.$ vs $3.30 \mathrm{~cm}^{2}$ [average of other morphology classes], $\left.\mathrm{p}=0.011\right)$. No association was found between LAA ostial area and LA sphericity, LAVI or LA fibrosis level.

LAA ostial parameters were also correlated with LA functional metrics. Total LAEF correlated negatively with LAA ostial area $(\mathrm{r}=-0.289, \mathrm{p}=0.0057)$. Indexed LAA ostial area was also negatively correlated with total LA strain $(\mathrm{r}=-0.248, \mathrm{p}=0.0185)$, and passive LA strain $(\mathrm{r}=-0.208, \mathrm{p}=0.049)$. There was a trend for negative correlation between LAA ostial and active LA strain, but this was not statistically significant $(\mathrm{p}=0.064)$.

Analysis results correlating LAA ostial parameters with LA morphological and functional markers are presented in Tables 3A/3B and 4, respectively. A selection of correlation analysis results from this analysis are also shown in Figure 2.

\section{Changes in Left Atrial Appendage Ostium Following Catheter Ablation}

A subgroup analysis on 37 patients who underwent pre- and post-ablation CMR studies was performed. On average, the post-ablation CMR was performed at $49.6+-26.7$ weeks after procedure day. The LAA 
ostial area reduced significantly from $3.84+-1.15 \mathrm{~cm}^{2}$ before ablation to $3.42+-0.96 \mathrm{~cm}^{2}$ after ablation $(\mathrm{p}=0.0004) .11$ of the 37 patients $(29.7 \%)$ experienced a reduction in ostial size to below $3.5 \mathrm{~cm}^{2}$ while the minor axis length of the LAA ostia decreased from $1.92 \mathrm{~cm}$ to $1.77 \mathrm{~cm}$ after ablation $(\mathrm{p}=0.0004)$, the major axis length did not demonstrate a significant reduction $(\mathrm{p}=0.1139)$. Other LA and LAA structural and functional markers were also examined in these patients and compared pre- and post-ablation. Other parameters such as LA volume, LAA volume, LAEF and various components of LA strain did not change significantly after ablation. This subgroup analysis on pre- and post-ablation changes is given in Table 5 .

Additional analysis was performed to examine LVEF change pre-/post-ablation and its association with LAA ostium area. First, LVEF increased from a pre-ablation average of $48.26 \%$ to a post-ablation average of $53.62 \%(\mathrm{p}=0.015)$. Correlation of pre-ablation LVEF and pre-ablation LAA ostium area showed a nearsignificant negative trend $(\mathrm{r}=-0.21, \mathrm{p}=0.083)$. There was no correlation between the post-ablation change in LVEF and the change in LAA ostium area $(\mathrm{p}=0.671)$. There was also no correlation between the postablation change in LVEF and the change in LAA ostium area $(\mathrm{p}=0.513)$. Results of this correlation analysis are presented in Figure 3.

\section{Discussion}

This is the first study to show that the LAA ostium reduces in size after AF ablation, and that this change is markedly asymmetrical, with the minor axis length shortening significantly more than the major axis length. In addition, we found that AF patients with HFrEF had a significantly greater LAA ostium areas than non-HFrEF patients. We also shown that LAA ostium size was positively correlated with both LA and LAA volume, and that patients with a "cauliflower" morphology of the LAA had significantly larger LAA ostia area than patients with other morphologies. Taking a functional perspective, the LAEF, as well as total and passive LA strains decreased as the LAA ostium size increased. LVEF increased significantly after ablation but changes in LVEF after ablation did not correlation with changes in LAA ostium area after ablation.

Of all the comorbidities analyzed, only HFrEF correlated significantly with LAA ostium size. This can be hypothesized to be due to stretching of the LAA ostium over time secondary to volume overload seen in heart failure(21). Both LA volume(22) and LAA volume(23) increases in patients with heart failure, and both LA enlargement(24) and LAA enlargement(25) is correlated with increased stroke risk. Another consideration originates from findings of the CASTLE-AF trial, which showed the superiority of ablation vs. medical therapy among AF patients with comorbid heart failure (22). The trial showed that the hazard ratio for stroke in these patients was 0.46 in favor of catheter ablation vs. medical therapy. This supports our hypothesis that by reducing the LAA ostial size, catheter ablation is also mitigating stroke risk in this patient group. Future investigation should further elucidate the underlying mechanisms for superiority of ablation when compared to medical therapy in the heart failure population.

When correlating the LAA ostium area to other morphological metrics of the LA and LAA, we found that the "cauliflower" LAA morphology was associated with the largest ostial area. Previous studies have found that "single-lobed", "cauliflower" LAA morphology and larger LAA volume was associated with higher rates of stroke $(26,27)$. This finding would be supported by the results of our investigation, as a larger LAA ostium may confer a greater risk for thromboemboli generated in the LAA of passing into systemic circulation (28). In addition, others have reported that non- "chicken-wing" LAA morphologies were associated with higher rates of stroke compared to the "chicken-wing" morphology (29). However, contradictory findings have also been reported, stating that stroke rates were similar across patients with different LAA morphologies (30). Though most studies including ours used a two-expert consensus in labeling LAA morphology, the relatively subjective nature of this categorization demands for future revamping and improvement.

In addition to anatomical metrics, various LA functional metrics were also found to be significantly associated with LAA ostium area. Specifically, the LAA ostium area was negatively correlated with LAEF, total LA strain and passive LA strain. While LAEF is more commonly used as a surrogate for LA function (31), the three components of LA strain may show a clearer picture and more thoroughly breakdown the role of the 
LAA during the normal cardiac cycle and in AF. The total, passive, and active components of LA strain correspond to the reservoir, conduit and pumping functions of the LA, respectively (32). Our finding that the LAA ostium area only correlates with total and passive LA strain may imply that the LAA is mostly involved in the reservoir and conduit roles of the atrium, but not necessarily in its pumping function. This is supported by previous investigations that have characterized the LAA's role as an overflow and decompression chamber for the LA (33). It is also unsurprising that the LAA does not contribute significantly to the LA pumping function, given its peripheral location, thin walls and relatively weak contractility (34).

Finally, our study is the first to characterize changes in the LAA ostium over time after AF ablation. Our hypothesis for the underlying mechanism includes an ablation-induced chronic remodeling of the left atrial substrate, which also includes changes to the LAA. The termination of AF and restoration of sinus rhythm may allow atrial myocytes to have more regular contractions and higher contractility(35). Additionally, although there was a near-significant negative correlation of pre-ablation LVEF and LAA ostium area, there was no direct correlation found between post-ablation change in LVEF and change in LAA ostium area. Furthermore, post-ablation change in LAEF also did not correlate with change in LAA ostium area. These findings suggest that the reduction in LAA ostium area seen after AF ablation is not a simple, direct result of functional improvement of the LA or LV. Rather, this reduction may be due to a more unique remodeling of the LAA in response to specifically catheter ablation therapy. Interestingly, previous studies have also found that a LAA ostium size $<3.5 \mathrm{~cm}^{2}$ was associated with a reduced risk of stroke(36). Although we did not find a direct correlation of LAA ostium area with stroke in our study cohort, $29.7 \%$ of our patients did experience a significant reduction of LAA ostia from $>3.5 \mathrm{~cm}^{2}$ to $<3.5 \mathrm{~cm}^{2}$. A prospective, longitudinal study is needed to track stroke incidence in this group.

Finally, the reduction in LAA ostium area observed in our patient cohort was markedly asymmetrical, with significantly more shrinkage in the minor axis length than the major axis length of the centroid. Without a larger and more comprehensive study, the clinical implications of this finding remain unclear. Although with the rising popularity of LAA occlusion devices such as the Watchman, it may be beneficial to take these post-ablation changes in the ostia into account when selecting the appropriate device size. It will also be intriguing to explore the underlying histology of LA myocytes surrounding the LAA ostium to determine whether distinct features are present in cells that align in the minor axis length direction. Lastly, it may be worth investigating patients whose LAA ostium does not shrink in size after ablation, as this cohort may exhibit an "ablation-refractory" phenotype and thus suffer from higher stroke risk despite ablation. For this patient cohort, LAA occlusion should be considered in addition to standard anticoagulation therapy to mitigate stroke risk (37).

Our findings bear important and compelling clinical implications that should be further explored in future investigations. Growing knowledge of the LAA and the LAA ostium is crucial to our overall understanding of $\mathrm{AF}$ pathophysiology and substrate stratification in $\mathrm{AF}$ management.

\section{Limitations:}

Our study has several limitations. First, this is a retrospective study that does not consider the implication of LAA ostial changes on clinical outcomes. Therefore, we are currently following our patients's cohort prospectively to assess for stroke incidence and rates of other complications. Second, only 37 patients in the cohort underwent post-ablation CMR studies and were thus used to calculate changes in LAA ostial size following catheter ablation. However, the reduction in ostial size was nevertheless significant in this subgroup.

\section{Conclusions}

After AF ablation, there is a significant asymmetrical reduction of the LAA ostial area that was independent of LVEF and LAEF changes. LAA ostial area was larger in patients with HFrEF, and in patients with "cauliflower" LAA morphology. Larger LAA ostial area was associated with greater LA and LAA volume, and with lower LAEF and LA strain. Remodeling of the LAA after AF ablation may help account for reduced risk of stroke and increased cardiac function. Future studies are needed to elucidate underlying 
mechanisms and explore clinical implications.

List of Abbreviations:

LA: Left Atrium

LAA: Left Atrial Appendage

CA: Catheter Ablation

AF: Atrial Fibrillation

LV: Left Ventricle

PVI: Pulmonary Vein Isolation

CMR: Cardiac Magnetic Resonance

BMI: Body Mass Index

LVEF: Left Ventricular Ejection Fraction

MRI: Magnetic Resonance Imaging

OAC: Oral Anticoagulants

MRA: Magnetic Resonance Angiography

PC: Phase Contrast

LGE: Late Gadolinium Enhancement

bSSFP: Balance Stead-State Free Precision

LAV: Left Atrial Volume

HFpEF: Heart Failure with Preserved Ejection Fraction

HFrEF: Heart Failure with Reduced Ejection Fraction

BSA: Body Surface Area

HTN: Hypertension

SSE: Stroke and Systemic Embolism

LAVI: Left Atrial Volume Index

LAEF: Left Atrial Ejection Fraction

Declarations:

Ethics Approval and Consent to Participate:

This study was reviewed and approved by the Tulane University School of Medicine Institutional Review Board (2019-1803-TUHSC). Consent to participate is not applicable.

\section{Consent for Publication:}

Not Applicable

\section{Availability of Data and Materials:}

The data that support the findings of this study are available from the corresponding author, NM, upon reasonable request.

Competing Interests: 
Dr. Marrouche reports receiving grant support and consulting fees from Abbott, Wavelet Health, Medtronic, Biosense Webster, Boston Scientific, GE Health Care, and Siemens, receiving consulting fees from Preventice, and holding equity in Marrek Inc. and Cardiac Design. Dr. Kholmovski reported receiving consulting fee from Marrek Inc. and holding equity in Marrek Inc. All other authors have no conflicts of interest associated with the content of this manuscript.

\section{Funding:}

Coherex contributed to the support of MRI analysists for this study.

\section{Authors' Contributions:}

YZ and NM established the study idea and designed the study. YZ, AHEH, ADP, MM, CN, LD, EK, RD, and TA collected data for analysis. $\mathrm{CH}, \mathrm{AM}, \mathrm{EK}$ and RD assisted with imaging acquisition and processing. CHL and CP performed statistical modeling and produced figures and tables. YZ, AHEH and CH drafted the initial version of the manuscript. All authors participated in revisions of the manuscript and approved it for submission and publication.

\section{Acknowledgements:}

We like to thank Ms. Agnieszka Jerzierska-Drutel, Dr. Arezu Bhatnagar, Mr. Charles Kilgore, Ms. Monique Young, Ms. Monica Smith, Ms. Noor Makan and other team members of the Tulane Heart and Vascular Institute for their contributions.

\section{Tables and Figures}

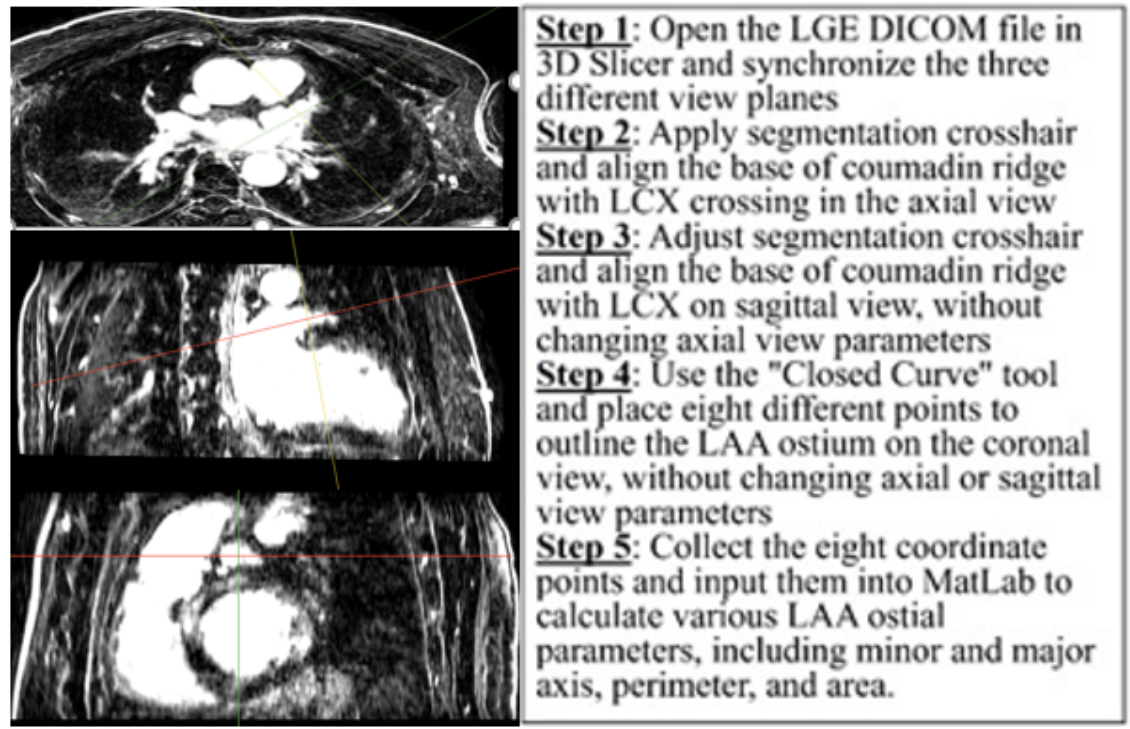

Figure 1 : Procedure for Measurement of the LA and LAA Ostium. (Abbreviations: LCX-Left Circumflex Artery) 

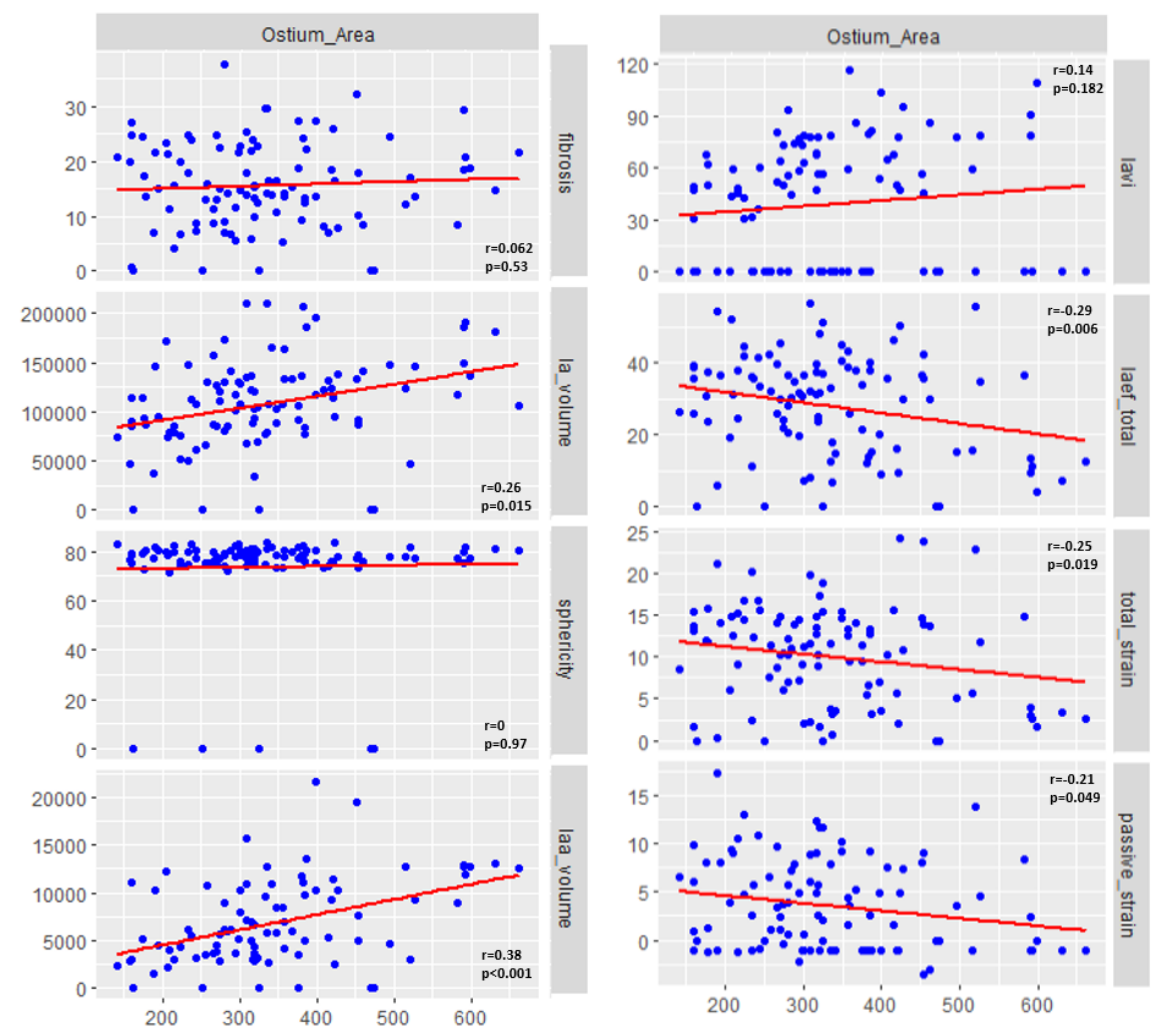

Figure 2: Correlation of Indexed LAA Ostium Area with various important LA and LAA morphological and functional metrics.

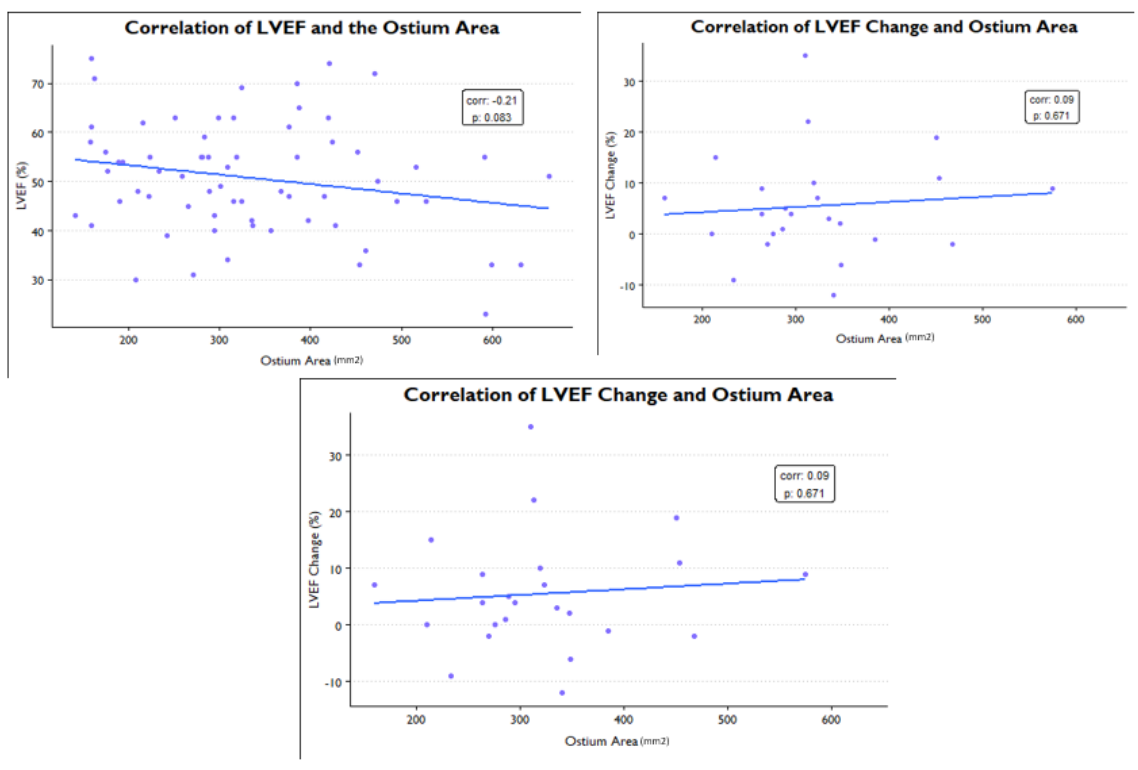

Figure 3: Correlation of LAA Ostium Area with LVEF.

Panel (A) shows the correlation of pre-ablation LAA ostium area with LVEF. Panel (B) shows the correlation 
of the change in LVEF after ablation with pre-ablation LAA ostium area. Panel (C) shows the correlation of the change in LVEF after ablation with the change in LAA ostium area after ablation.

\begin{tabular}{|c|c|c|c|}
\hline$N=101$ & Average & & Average \\
\hline Age (years) & $60.1+/-11.1$ & LAVI $\left(\mathrm{mL} / \mathrm{m}^{2}\right)$ & $38.66+/-10.2$ \\
\hline Male (\% of total) & $70(69.3 \%)$ & LA Sphericity Index & $73.6+/-18.9$ \\
\hline Female ( $\%$ of total) & $31(30.7 \%)$ & LAA Volume $(\mathrm{mL})$ & $6.76+/-4.47$ \\
\hline BMI & $29.22+/-5.08$ & Fibrosis (\% of LA wall) & $15.51+/-8.02$ \\
\hline $\operatorname{BSA}\left(\mathrm{m}^{2}\right)$ & $1.83+/-0.68$ & LAA Ostium Area $\left(\mathrm{cm}^{2}\right)$ & $3.32+1.17$ \\
\hline HFrEF ( $\%$ of total) & $24(23.8 \%)$ & Indexed LAA Ostium Area $\left(\mathrm{cm}^{2} / \mathrm{m}^{2}\right)$ & $1.67+/-0.56$ \\
\hline $\mathrm{HFpEF}$ (\% of total) & $7(6.9 \%)$ & & \\
\hline $\operatorname{LVEF}(\%)$ & $48.85+/-12.5$ & & \\
\hline HTN (\% of total) & $47(46.5 \%)$ & LAA Ostial Major Axis Length (cm) & $2.44+/-0.48$ \\
\hline $\mathrm{DM}(\%$ of total $)$ & $12(11.9 \%)$ & LAA Ostial Minor Axis Length (cm) & $1.72+/-0.34$ \\
\hline Stroke/SSE (\% of total) & $8(7.9 \%)$ & LAA Ostial Perimeter $(\mathrm{cm})$ & $6.35+/-1.17$ \\
\hline \multirow[t]{5}{*}{ Smoking (\% of total) } & $22(21.8 \%)$ & LAA Morphology Type & \\
\hline & & Chicken Wing (\% of total) & $78(77.2 \%)$ \\
\hline & & Cauliflower (\% of total) & $10(9.9 \%)$ \\
\hline & & Windsock (\% of total) & $7(6.93 \%)$ \\
\hline & & Cactus (\% of total) & $6(5.94 \%)$ \\
\hline Hx of Cardioversion (\% of total) & $34(33.7 \%)$ & Failed Trial of Antiarrhythmics ( $\%$ of total) & $39(38.6 \%)$ \\
\hline
\end{tabular}

Table 1: Baseline Characteristics of the Study Population.

\begin{tabular}{|c|c|c|c|c|c|}
\hline & $\mathrm{N}$ & LAA Ostium Area $\left(\mathrm{mm}^{2}\right)$ & $\mathrm{p}$-value & Indexed LAA Ostium Area $\left(\mathrm{mm}^{2} / \mathrm{m}^{2}\right)$ & $\mathrm{p}$-value \\
\hline HFrEF & 24 & $388 \pm 110.85$ & 0.0148 & $189.67 \pm 56.65$ & 0.0268 \\
\hline No HFrEF & 77 & $319.83 \pm 115.44$ & & $160.22 \pm 189.67$ & \\
\hline HTN & 47 & $347.19 \pm 115.79$ & 0.2664 & $167.85 \pm 60.58$ & 1 \\
\hline No HTN & 54 & $322.67 \pm 118.57$ & & $166.26 \pm 51.58$ & \\
\hline DM & 12 & $363.75 \pm 129.85$ & 0.3843 & $175.79 \pm 63.6$ & 0.6152 \\
\hline No DM & 89 & $330.23 \pm 115.71$ & & $165.75 \pm 55.25$ & \\
\hline Stroke/SSE & 8 & $353 \pm 207.97$ & 0.5561 & $174.33 \pm 92.74$ & 0.6360 \\
\hline No Stroke/SSE & 93 & $332.65 \pm 107.64$ & & $166.38 \pm 52.11$ & \\
\hline
\end{tabular}

Table 2 : Correlation of LAA Ostium Area and Preexisting Comorbidities/Risk Factors.

\begin{tabular}{lll}
\hline & Correlation & p-value \\
\hline LAA Ostium Area $\left(\mathrm{mm}^{2}\right)$ with Fibrosis & 0.06226 & 0.5383 \\
Indexed LAA Ostium Area $\left(\mathrm{mm}^{2} / \mathrm{m}^{2}\right)$ with Fibrosis & 0.02149 & 0.8407 \\
LAA Ostium Area $\left(\mathrm{mm}^{2}\right)$ with LA Volume & 0.31034 & $\mathbf{0 . 0 0 1 7}$ \\
Indexed LAA Ostium Area $\left(\mathrm{mm}^{2} / \mathrm{m}^{2}\right)$ with LA Volume & 0.25668 & $\mathbf{0 . 0 1 4 6}$ \\
LAA Ostium Area $\left(\mathrm{mm}^{2}\right)$ with $\left.\mathrm{Sphhricity}^{2} / \mathrm{m}^{2}\right)$ with Sphericity & 0.02697 & 0.79 \\
Indexed LAA Ostium Area $\left(\mathrm{mm}^{2} / 0.00345\right.$ & 0.9743 \\
LAA Ostium Area $\left(\mathrm{mm}^{2}\right)$ with $\mathrm{LAA}$ Volume & 0.42015 & $<\mathbf{0 . 0 0 0 1}$ \\
Indexed LAA Ostium Area $\left(\mathrm{mm}^{2} / \mathrm{m}^{2}\right)$ with LAA Volume & 0.37534 & $\mathbf{0 . 0 0 0 7}$ \\
LAA Ostium Area $\left(\mathrm{mm}^{2}\right)$ with LAVI & 0.10935 & 0.2813 \\
Indexed LAA Ostium Area $\left(\mathrm{mm}^{2} / \mathrm{m}^{2}\right)$ with LAVI & 0.14194 & 0.182 \\
\hline
\end{tabular}


Table 3A : Correlation of LAA Ostial Parameters with LA and LAA Structural Markers

\begin{tabular}{lllll}
\hline LAA Morphology Type & LAA Ostium Area $\left(\mathrm{mm}^{2}\right)$ & p-value & Indexed LAA Ostium Area $\left(\mathrm{mm}^{2} / \mathrm{m}^{2}\right)$ & p-value \\
\hline Chicken Wing & $340.27+/-109.75$ & $\mathbf{0 . 0 1 1 1}$ & $167.71+/-50.81$ & $\mathbf{0 . 0 1 8 9}$ \\
Cauliflower & $433.88+/-138.97$ & & $224.35+/-70.86$ & \\
Windsock & $234.5+/-69.19$ & $109.28+/-21.41$ & \\
Cactus & $322.33+/-126.79$ & $171.46+/-66.04$ & \\
\hline
\end{tabular}

Table 3B : Correlation of LAA Ostial Parameters with LAA Morphology Type $\backslash$

\begin{tabular}{lll}
\hline & Correlation & p-value \\
\hline LAA Ostium Area $\left(\mathrm{mm}^{2}\right)$ with LAEF & -0.23779 & $\mathbf{0 . 0 1 7 2}$ \\
Indexed LAA Ostium Area $\left(\mathrm{mm}^{2} / \mathrm{m}^{2}\right)$ with LAEF & -0.28898 & $\mathbf{0 . 0 0 5 7}$ \\
LAA Ostium Area $\left(\mathrm{mm}^{2}\right)$ with LA Strain (Total) & -0.18042 & 0.0724 \\
Indexed LAA Ostium Area $\left(\mathrm{mm}^{2} / \mathrm{m}^{2}\right)$ with LA Strain (Total) & -0.24795 & $\mathbf{0 . 0 1 8 5}$ \\
LAA Ostium Area $\left(\mathrm{mm}^{2}\right)$ with LA Strain (Passive) & -0.20025 & $\mathbf{0 . 0 4 5 8}$ \\
Indexed LAA Ostium Area $\left(\mathrm{mm}^{2} / \mathrm{m}^{2}\right)$ with LA Strain (Passive) & 0.2081 & $\mathbf{0 . 0 4 9}$ \\
LAA Ostium Area $\left(\mathrm{mm}^{2}\right)$ with LA Strain (Active) & -0.17137 & 0.0882 \\
Indexed LAA Ostium Area $\left(\mathrm{mm}^{2} / \mathrm{m}^{2}\right)$ with LA Strain (Active) & -0.19606 & 0.064 \\
\hline
\end{tabular}

Table 4: Correlation of LAA Ostial Parameters with LA Functional Markers

\begin{tabular}{llll}
\hline Parameter $(\mathrm{N}=37)$ & Pre-Ablation & Post-Ablation & p-value \\
\hline LAA Ostium Area $\left(\mathrm{mm}^{2}\right)$ & $383.89+/-114.84$ & $342.35+/-95.71$ & $\mathbf{0 . 0 0 0 4}$ \\
LVEF $(\%)$ & $48.26+/-9.02$ & $53.62+/-8.82$ & $\mathbf{0 . 0 1 5}$ \\
LAA Ostium Minor Axis Length $(\mathrm{cm})$ & $19.17+/-3.24$ & $17.71+/-3.03$ & $\mathbf{0 . 0 0 0 4}$ \\
LAVI $\left(\mathrm{mL} / \mathrm{m}^{2}\right)$ & $85.36+/-19.55$ & $73.67+/-12.05$ & 0.2204 \\
LAA Volume $(\mathrm{mL})$ & $8.21+/-5.32$ & $7.82+/-4.01$ & 0.9483 \\
LAEF Total $(\%)$ & $25.82+/-14.06$ & $25.96+/-8.18$ & 0.9191 \\
LA Fibrosis $(\%)$ & $18.45+/-6.24$ & $13.71+/-6.75$ & 0.0783 \\
LA Strain Total $\left(\mathrm{s}^{-1}\right)$ & $9.51+/-4.53$ & $8.19+/-2.54$ & 0.4584 \\
\hline
\end{tabular}

Table 5: Post-Ablation Changes in LA and LAA Morphological and Functional Parameters.

\section{References}

1. Nattel S. New ideas about atrial fibrillation 50 years on. Nature. 2002;415(6868):219-26.

2. Nattel S, Burstein B, Dobrev D. Atrial remodeling and atrial fibrillation: mechanisms and implications. Circulation: Arrhythmia and Electrophysiology. 2008;1(1):62-73.

3. Lip GY, Tse H-F. Management of atrial fibrillation. The Lancet. 2007;370(9587):604-18.

4. Arboix A, Alió J. Cardioembolic stroke: clinical features, specific cardiac disorders and prognosis. Curr Cardiol Rev. 2010;6(3):150-61.

5. Association DwtscotEHR, Surgery EbtEAfC-T, Members ATF, Camm AJ, Kirchhof P, Lip GY, et al. Guidelines for the management of atrial fibrillation: the Task Force for the Management of Atrial Fibrillation of the European Society of Cardiology (ESC). European heart journal. 2010;31(19):2369-429. 
6. A Comparison of Rate Control and Rhythm Control in Patients with Atrial Fibrillation. New England Journal of Medicine. 2002;347(23):1825-33.

7. Kirchhof P, Camm AJ, Goette A, Brandes A, Eckardt L, Elvan A, et al. Early Rhythm-Control Therapy in Patients with Atrial Fibrillation. New England Journal of Medicine. 2020;383(14):1305-16.

8. Andrade JG, Wells GA, Deyell MW, Bennett M, Essebag V, Champagne J, et al. Cryoablation or Drug Therapy for Initial Treatment of Atrial Fibrillation. New England Journal of Medicine. 2020;384(4):305-15.

9. Marrouche NF, Brachmann J, Andresen D, Siebels J, Boersma L, Jordaens L, et al. Catheter ablation for atrial fibrillation with heart failure. New England Journal of Medicine. 2018;378(5):417-27.

10. Blomström-Lundqvist C, Gizurarson S, Schwieler J, Jensen SM, Bergfeldt L, Kennebäck G, et al. Effect of catheter ablation vs antiarrhythmic medication on quality of life in patients with atrial fibrillation: the CAPTAF randomized clinical trial. Jama. 2019;321(11):1059-68.

11. Nademanee K, McKenzie J, Kosar E, Schwab M, Sunsaneewitayakul B, Vasavakul T, et al. A new approach for catheter ablation of atrial fibrillation: mapping of the electrophysiologic substrate. Journal of the American College of Cardiology. 2004;43(11):2044-53.

12. Spencer K, Mor-Avi V, Gorcsan Jr, DeMaria A, Kimball T, Monaghan M, et al. Effects of aging on left atrial reservoir, conduit, and booster pump function: a multi-institution acoustic quantification study. Heart. 2001;85(3):272-7.

13. Heppell R, Berkin K, McLenachan J, Davies J. Haemostatic and haemodynamic abnormalities associated with left atrial thrombosis in non-rheumatic atrial fibrillation. Heart. 1997;77(5):407-11.

14. Al-Saady N, Obel O, Camm A. Left atrial appendage: structure, function, and role in thromboembolism. Heart. 1999;82(5):547-54.

15. Barbero U, Ho SY. Anatomy of the atria. Herzschrittmachertherapie + Elektrophysiologie. 2017;28(4):347-54.

16. Wang Y, Di Biase L, Horton RP, Nguyen T, Morhanty P, Natale A. Left atrial appendage studied by computed tomography to help planning for appendage closure device placement. Journal of cardiovascular electrophysiology. 2010;21(9):973-82.

17. Chava R, Turagam MK, Lakkireddy DD. Left Atrial Appendage Occlusion: What Are the Options and Where is the Evidence? J Innov Card Rhythm Manag. 2018;9(4):3095-106.

18. Marrouche NF, Greene T, Dean JM, Kholmovski EG, Boer LMd, Mansour M, et al. Efficacy of LGEMRI-guided fibrosis ablation versus conventional catheter ablation of atrial fibrillation: The DECAAF II trial: Study design. Journal of cardiovascular electrophysiology. 2021;32(4):916-24.

19. Marrouche NF, Wilber D, Hindricks G, Jais P, Akoum N, Marchlinski F, et al. Association of Atrial Tissue Fibrosis Identified by Delayed Enhancement MRI and Atrial Fibrillation Catheter Ablation: The DECAAF Study. JAMA. 2014;311(5):498-506.

20. Masci A, Barone L, Dede L, Fedele M, Tomasi C, Quarteroni A, et al. The Impact of Left Atrium Appendage Morphology on Stroke Risk Assessment in Atrial Fibrillation: A Computational Fluid Dynamics Study. Frontiers in Physiology. 2019;9(1938).

21. Takemoto Y, Barnes ME, Seward JB, Lester SJ, Appleton CA, Gersh BJ, et al. Usefulness of Left Atrial Volume in Predicting First Congestive Heart Failure in Patients [?]65 Years of Age With Well-Preserved Left Ventricular Systolic Function. The American Journal of Cardiology. 2005;96(6):832-6.

22. Dernellis JM, Stefanadis CI, Zacharoulis AA, Toutouzas PK. Left atrial mechanical adaptation to long-standing hemodynamic loads based on pressure-volume relations. The American journal of cardiology. 1998;81(9):1138-43. 
23. Bakalli A, Kamberi L, Pllana E, Zahiti B, Dragusha G, Brovina A. The influence of left ventricular diameter on left atrial appendage size and thrombus formation in patients with dilated cardiomyopathy. Turk Kardiyol Dern Ars. 2010;38(2):90-4.

24. Xu Y, Zhao L, Zhang L, Han Y, Wang P, Yu S. Left Atrial Enlargement and the Risk of Stroke: A Meta-Analysis of Prospective Cohort Studies. Front Neurol. 2020;11:26-.

25. Taina M, Vanninen R, Hedman M, Jakala P, Karkkainen S, Tapiola T, et al. Left Atrial Appendage Volume Increased in More Than Half of Patients with Cryptogenic Stroke. PLOS ONE. 2013;8(11):e79519.

26. Di Biase L, Santangeli P, Anselmino M, Mohanty P, Salvetti I, Gili S, et al. Does the left atrial appendage morphology correlate with the risk of stroke in patients with atrial fibrillation? Results from a multicenter study. Journal of the American College of Cardiology. 2012;60(6):531-8.

27. Korhonen M, Muuronen A, Arponen O, Mustonen P, Hedman M, Jakala P, et al. Left atrial appendage morphology in patients with suspected cardiogenic stroke without known atrial fibrillation. PLoS One. 2015;10(3):e0118822.

28. Kimura T, Takatsuki S, Inagawa K, Katsumata Y, Nishiyama T, Nishiyama N, et al. Anatomical characteristics of the left atrial appendage in cardiogenic stroke with low CHADS2 scores. Heart Rhythm. 2013;10(6):921-5.

29. Lupercio F, Ruiz JC, Briceno DF, Romero J, Villablanca PA, Berardi C, et al. Left atrial appendage morphology assessment for risk stratification of embolic stroke in patients with atrial fibrillation: a metaanalysis. Heart Rhythm. 2016;13(7):1402-9.

30. Khurram IM, Dewire J, Mager M, Maqbool F, Zimmerman SL, Zipunnikov V, et al. Relationship between left atrial appendage morphology and stroke in patients with atrial fibrillation. Heart rhythm. 2013;10(12):1843-9.

31. Appleton CP, Galloway JM, Gonzalez MS, Gaballa M, Basnight MA. Estimation of left ventricular filling pressures using two-dimensional and Doppler echocardiography in adult patients with cardiac disease: additional value of analyzing left atrial size, left atrial ejection fraction and the difference in duration of pulmonary venous and mitral flow velocity at atrial contraction. Journal of the American College of Cardiology. $1993 ; 22(7): 1972-82$.

32. Yamada A, Hashimoto N, Fujito H, Hatta T, Saito Y, Otsuka N, et al. Comprehensive assessment of left atrial and ventricular remodeling in paroxysmal atrial fibrillation by the cardiovascular magnetic resonance myocardial extracellular volume fraction and feature tracking strain. Scientific reports. 2021;11(1):1-12.

33. Donal E, Yamada H, Leclercq C, Herpin D. The left atrial appendage, a small, blind-ended structure: a review of its echocardiographic evaluation and its clinical role. Chest. 2005;128(3):1853-62.

34. Beigel R, Wunderlich NC, Ho SY, Arsanjani R, Siegel RJ. The left atrial appendage: anatomy, function, and noninvasive evaluation. JACC: Cardiovascular imaging. 2014;7(12):1251-65.

35. Cha Y-M, Redfield MM, Shen W-K, Gersh BJ. Atrial Fibrillation and Ventricular Dysfunction. Circulation. 2004;109(23):2839-43.

36. Lee JM, Shim J, Uhm J-S, Kim YJ, Lee H-J, Pak H-N, et al. Impact of Increased Orifice Size and Decreased Flow Velocity of Left Atrial Appendage on Stroke in Nonvalvular Atrial Fibrillation. American Journal of Cardiology. 2014;113(6):963-9.

37. Syed FF, DeSimone CV, Friedman PA, Asirvatham SJ. Left atrial appendage exclusion for atrial fibrillation. Cardiol Clin. 2014;32(4):601-25. 